Recherches en didactique des langues et des cultures

Les cahiers de l'Acedle

6-1 | 2009

L'Alsace au cœur du plurilinguisme (2)

\title{
La sensibilisation à l'interculturel dans l'enseignement de l'anglais à l'école primaire : observations en CE1 et CE2
}

\section{Michèle Catroux}

\section{Q OpenEdition}

1 Journals

Édition électronique

URL : http://journals.openedition.org/rdlc/2045

DOI : $10.4000 /$ rdlc. 2045

ISSN : 1958-5772

Éditeur

ACEDLE

Référence électronique

Michèle Catroux, « La sensibilisation à l'interculturel dans l'enseignement de l'anglais à l'école primaire : observations en CE1 et CE2 ", Recherches en didactique des langues et des cultures [En ligne], 6-1 | 2009, mis en ligne le 01 avril 2009, consulté le 24 avril 2019. URL : http:// journals.openedition.org/rdlc/2045; DOI : 10.4000/rdlc.2045

Ce document a été généré automatiquement le 24 avril 2019

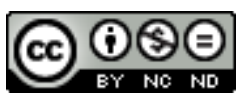

Recherches en didactique des langues et des cultures is licensed under a Creative Commons AttributionNonCommercial-NoDerivatives 4.0 International License 


\title{
La sensibilisation à l'interculturel dans l'enseignement de l'anglais à l'école primaire : observations en CE1 et CE2
}

\author{
Michèle Catroux
}

\section{Introduction}

1 La politique linguistique des institutions européennes se prononce en faveur du plurilinguisme afin de répondre aux besoins individuels et sociaux d'une Europe où les mouvements migratoires s'intensifient. Dans cette optique, le Cadre Européen Commun de Référence pour les Langues met en place les principes d'une diversification linguistique et culturelle. Le Cadre définit une compétence à la fois plurilingue et pluriculturelle comme une "compétence à communiquer langagièrement et à interagir culturellement d'un acteur qui possède, à des degrés divers, la maîtrise de plusieurs langues et l'expérience de plusieurs cultures" (CECRL, 2001: 129). Ces attentes conduisent les acteurs des systèmes éducatifs à s'interroger sur les changements didactiques des langues / cultures qui doivent accompagner la construction d'une telle compétence et en particulier dans le champ de l'enseignement / apprentissage des langues étrangères à l'école primaire.

2 Dans cette perspective, nous avons mis en place des moments de sensibilisation à l'interculturel dans deux classes de CE1 et de CE2 en partenariat avec leurs enseignantes, avant même de démarrer l'enseignement linguistique proprement dit pour favoriser une démarche de prise de conscience de la diversité culturelle. Les premières leçons de l'année ont été conçues afin de faire émerger les représentations des élèves sur la nature et les modalités d'usage des langues étrangères. Elles ont été suivies d'une réflexion sur les différentes sonorités des langues et l'existence de divers alphabets. Les enregistrements audio des interactions élèves-enseignante et élèves-élèves ont ensuite 
été analysés pour tenter de reconnaître les compétences mises en jeu. Des exemples tirés de ces enregistrements nous conduiront à nous interroger sur les atouts liés à cette nouvelle expérience d'apprentissage pour les élèves ainsi que sur les obstacles identifiés au cours de cette première phase d'expérimentation.

\section{De l'acquisition de connaissances culturelles à la construction de la compétence interculturelle}

\subsection{L'enseignement de la "culture"}

3 Avant d'exposer les modalités de ce travail, il convient de préciser la notion de compétence interculturelle en l'opposant tout d'abord à celle d'enseignement de faits culturels communément admise en milieu scolaire.

4 Les volets "culture" et "langue" ont souvent été considérés comme deux volets distincts dont l'apprentissage pouvait se faire isolément. La finalité de l'enseignement de la langue étrangère s'est trouvée être essentiellement la transmission de connaissances parmi lesquelles figuraient les "faits culturels". L'unanimité se fait actuellement sur l'indispensable place que la culture doit occuper dans l'enseignement et l'apprentissage des langues étrangères.

On s'accorde à affirmer avec Galisson et Puren (1999 : 96) que si :

le commun des mortels n'apprend pas une langue pour en démonter les mécanismes et manipuler gratuitement des mots nouveaux, mais pour fonctionner dans la culture qui va avec cette langue, on aboutit à la conclusion que celle-ci n'est pas une fin en soi, mais un moyen pour opérer culturellement, pour comprendre et produire du sens, avec les outils et dans l'univers de l'Autre. Donc que la culture, en tant qu'au-delà de la langue, est la fin recherchée.

6 Néanmoins, si la nécessité d'un apport culturel dans le contenu de séquences d'apprentissage se trouve affirmée, tant dans les programmes officiels que dans les discours savants, cette nécessité soulève aussitôt une série de questions : quelle culture enseigner, comment et quels en sont les véritables enjeux ?

7 Les programmes d'enseignement de l'école primaire (B.O. hors série $\mathrm{n}^{\circ} 1 \mathrm{du}$ 14 février 2002) limitent la dimension culturelle de l'enseignement des langues à la transmission de :

connaissances sur les modes de vie des locuteurs de la langue apprises [...] à l'occasion des activités de langue organisées autour de thématiques liées à l'environnement d'élèves de cet âge : la vie en famille, l'habitation, l'école, les amis et les loisirs, les animaux familiers, les rythmes de l'année (anniversaire, fêtes). Ainsi, l'élève découvre la vie scolaire d'enfants du même âge, notamment grâce à l'observation de matériaux (audio)visuels.

Les finalités d'une approche culturelle ne sont pas clairement explicitées et il n'existe pas de guidage précis quant à l'opérationnalisation de ces objectifs. Faute de réponse adéquate, les enseignants tentent d'"enseigner la culture", ou encore "la civilisation", en offrant un catalogue d'informations parcellaires, une présentation décontextualisée de cartes postales conduisant le plus souvent à renforcer des stéréotypes mal dissimulés. C'est ce qu'Abdallah-Pretceille (1999:82) appelle la "folklorisation des cultures". Les contenus ont souvent été réduits à leurs manifestations les plus facilement perceptibles 
(cuisine, artisanat, festivals ...) et se sont trouvés enfermés dans des descriptions intemporelles et généralisantes, vidés de leur dynamique et décontextualisés.

D'autre part, la définition de la "culture anglophone" a subi ces dernières années des mutations importantes. La diversification du "monde anglophone" ne cesse de dérouter l'enseignant d'anglais en lui conférant une latitude déconcertante quant à la sélection de faits représentatifs de cette culture. De plus, comme le souligne Abdallah-Pretceille (1996:73-74), les connaissances culturelles n'améliorent pas nécessairement la compréhension. Au contraire, elles peuvent servir d'écran car aucun individu n'est le représentant de son groupe. Une stratégie plus englobante et plus dynamique permettrait non pas d'accumuler des connaissances relatives à un groupe social de référence dominant, mais bien plutôt de procéder à une "analyse des situations". La notion de compétence interculturelle peut alors se substituer à celle de culture.

\subsection{La construction d'une compétence interculturelle}

10 Le Cadre Européen (2001 : 83-84) définit la compétence interculturelle comme :

la connaissance, la conscience et la compréhension des relations, (ressemblances et différences distinctives) entre "le monde d'où l'on vient" et "le monde de la communauté cible" sont à l'origine d'une prise de conscience interculturelle. Il faut souligner que la prise de conscience interculturelle inclut la conscience de la diversité régionale et sociale des deux mondes.

Les aptitudes et les savoir-faire interculturels se déclinent comme :

- la capacité d'établir une relation entre la culture d'origine et la culture étrangère ;

- la sensibilisation à la notion de culture et la capacité de reconnaître et d'utiliser des stratégies variées pour établir le contact avec des gens d'une autre culture ;

- la capacité de jouer le rôle d'intermédiaire culturel entre sa propre culture et la culture étrangère et de gérer efficacement des situations de malentendus et de conflits culturels ;

- la capacité à aller au-delà de relations superficielles stéréotypées.

12 Avec la notion d'acteur social, le Cadre Européen fournit les éléments d'un cadre didactique à l'intérieur duquel peuvent se développer les compétences interculturelles. Il va s'agir de permettre un dialogue entre des individus ayant des identités sociales différentes, et de favoriser les interactions entre ces acteurs. L'apprenant se trouvera à la fois représentant de sa culture d'origine, intermédiaire culturel entre les communautés dans lesquelles il agit, et au contact avec un étranger dont il devra découvrir les rituels sociaux.

\subsection{La notion d"'intermédiaire interculturel"}

13 L'objectif de l'enseignement / apprentissage des langues / cultures devient un accès à l'altérité tel que le préconisait le rapport Bourdieu-Gros en 1989. Le Cadre Européen de Référence donne toute sa force à ce concept en l'inscrivant dans une "perspective actionnelle" qui :

...considère avant tout l'usager et l'apprenant d'une langue comme des acteurs sociaux ayant à accomplir des tâches (qui ne sont pas seulement langagières) dans des circonstances et un environnement donnés, à l'intérieur d'un domaine d'action particulier. Si les actes de parole se réalisent dans une activité langagière, celles-ci s'inscrivent elles-mêmes à l'intérieur d'actions en contexte social qui seules leur donnent leur pleine signification (CECRL, $2001: 15)$. 

découverte et à la mise en interaction avec les autres cultures. Byram (1997:49-54) complète ces quatre compétences par le savoir-s'engager qui confère une dimension politique, éducative et critique à la compétence interculturelle.

\section{Questions de recherche et adaptation du modèle}

\subsection{Identification des contraintes}

21 On le voit, il est nécessaire de procéder à un changement d'orientation dans l'enseignement de la culture : l'objectif n'est pas d'acquérir des connaissances sur les autres ou d'adopter certains comportements, mais de développer la capacité à comprendre et à communiquer avec les autres. Comment pouvons-nous adapter ce projet à des apprenants débutants en langue étrangère mais également au tout début de leur propre développement à la fois psychologique, social et cognitif ? 

différente ou à identifier les relations entre des documents de différents pays est étroitement liée à la connaissance que l'apprenant a de son environnement et de l'environnement autre. Si l'on considère avec Byram (1997:37) que la capacité d'interprétation et de mise en relation ne peut exister que sur la base d'une connaissance déjà développée, la question se pose de savoir si des enfants en développement cognitif et social comme les élèves d'école primaire peuvent développer ce genre de capacités. peuvent faire craindre que l'enfant ne possède pas la possibilité de procéder à des transferts de la langue maternelle comme l'adulte dont les capacités métalinguistiques et métacognitives sont plus grandes. Il peut donc moins bien utiliser ses connaissances antérieures et utiliser des stratégies cognitives moins efficaces dans le domaine de la conceptualisation et de la mémorisation, entre autres (Pross et al., 2002). Cependant, dans des cas d'apprentissage très précoce, on constate généralement que l'apprentissage se fait

Recherches en didactique des langues et des cultures, 6-1 | 2009 
avec facilité et avec beaucoup de plaisir, ce qui permet de créer une attitude globalement positive à l'égard de la langue apprise en contexte scolaire. Les facteurs favorables à une telle formation relèvent des caractéristiques d'adaptabilité et d'absence d'anxiété devant l'inconnu que l'on peut voir chez les jeunes enfants.

Rappelons enfin que l'enseignement de la langue étrangère passe par une pratique essentiellement orale et que de toute façon, les enfants sont non-lecteurs de la L2 à ce stade de leur apprentissage. Comment alors utiliser des documents culturels écrits qui ne feront pas appel à la lecture?

\subsection{Conception de la démarche interculturelle}

Afin de constituer notre propre modèle de référence, nos emprunts à des programmes existants sont nombreux. Le Programme Evlang a été notre source d'inspiration primordiale. Programme européen d'innovation pédagogique et de recherche entre 1997 et 2001, il a réuni des partenaires de six pays (Autriche, Espagne, Italie, France et Suisse). L'objectif du programme Evlang était de vérifier si les activités d'éveil aux langues à l'école primaire conduisaient bien aux effets espérés. Les buts étaient :

- le développement de représentations et d'attitudes positives par rapport à la diversité linguistique et culturelle et de motivation pour l'apprentissage des langues ;

- le développement d'aptitudes d'ordre métalinguistique/méta-communicatif et le développement d'une culture linguistique.

De même, le programme Janua Linguarum en 2003 s'est concentré sur la diffusion de l'éveil aux langues dans seize pays d'Europe et sur l'étude des conditions de son introduction dans les programmes. C'est à partir des supports conçus pour la réalisation de ces programmes que nous avons mis en œuvre une "démarche interculturelle", terme que nous empruntons à Auger (2005).

\subsection{Protocole d'observation}

31 Compte tenu des contraintes identifiées préalablement, nous avons mis en œuvre des séances de sensibilisation à l'interculturel dans deux classes de CE1 et de CE2 en accord avec leurs enseignantes avant les premières leçons d'anglais. Notre objectif est de favoriser une réflexion, une prise de conscience de la diversité linguistique à travers des activités de découverte des langues. La première phase d'expérimentation se centre essentiellement sur des phénomènes linguistiques. Nous introduirons des éléments de type civilisationnel après que l'enseignement de la langue aura commencé afin de donner un substrat plus conséquent à cette sensibilisation.

Les séances sont conduites en français, choix indiscutable à ce stade de l'apprentissage. Comme il s'agit d'une mise en perspective de la langue / culture autre d'abord et anglophone ensuite, les séances se placent en amont de l'apprentissage de la langue étrangère. Les enfants ne sont pas soumis à une quelconque production évaluée mais mis en contact avec des documents leur permettant de créer des questionnements et d'émettre des hypothèses sur les langues étrangères. Dans ce but, ils sont amenés à puiser dans leur patrimoine culturel en fonction de leurs origines familiales et à faire émerger leurs connaissances latentes. 
33 En accord avec la grille de compétences établie par Byram (1998), nous avons établi quatre types de comportements observables :

- nous tenterons d'identifier des traces de développement de savoir-être. Il s'agira de relever dans les interactions une posture d'ouverture à la langue autre, et un désir de réflexion sur la nature des langues en général et sur la langue étrangère en particulier ;

- nous porterons notre attention sur l'existence de savoir-faire et savoir-comprendre qui mobilisent les habiletés à comprendre les faits linguistiques et culturels nouveaux. À cet égard, il sera nécessaire de discerner les stratégies de compréhension mises en œuvre spontanément par les élèves ;

- les savoirs seront mesurés en terme de patrimoine culturel sous-jacent et nous verrons quelles sont les connaissances auxquelles les élèves font appel pour construire une compétence interculturelle ;

- les savoir-apprendre seront reconnus comme la capacité à établir des liens entre les savoirs existant dans le patrimoine des élèves et les nouveaux savoirs auxquels ils sont confrontés.

Le savoir-s'engager ne nous paraît pas pertinent à ce stade de l'apprentissage et ne fera donc pas l'objet de critères d'observation.

L'objectif est de trouver des éléments de réponses aux deux questions suivantes :

- les séances permettent-elles l'ébauche d'un éveil à l'interculturel?

- cette démarche est-elle pertinente au niveau du CE1 et du CE2 ?

\subsection{Séances observées}

Les séances ont été mises en œuvre à partir des documents présentés dans l'ouvrage "Les langues du monde au quotidien" du cycle 2 et du cycle 3 (Kervran, 2006). Les tableaux cidessous présentent l'ensemble du dispositif :

Tableau 1 : séances $\mathrm{CE} 1$.

\begin{tabular}{|l|l|l|}
\hline $\begin{array}{l}\text { Contenu des } \\
\text { séances CE1 }\end{array}$ & $\begin{array}{l}\text { Activité des élèves } \\
\text { (9 filles et 14 garçons) }\end{array}$ & Objectif de la séance \\
\hline Séance 1 & $\begin{array}{l}\text { répondre à la question "À quoi sert une } \\
\text { langue ?" } \\
\text { nommer des langues étrangères }\end{array}$ & $\begin{array}{l}\text { lane langue, et de la diversité des } \\
\text { langues connues }\end{array}$ \\
\hline Séance 2 & $\begin{array}{l}\text { compléter les marguerites des langues avec } \\
\text { les mots que l'on connaît dans une langue }\end{array}$ & $\begin{array}{l}\text { prendre conscience des mots que } \\
\text { l'on connaît en langue étrangère }\end{array}$ \\
\hline Séance 3 & $\begin{array}{l}\text { langues (français, une langue alphabétique } \\
\text { classer les jours de la semaine en trois }\end{array}$ & $\begin{array}{l}\text { développer une stratégie de } \\
\text { déduction et d'inférence }\end{array}$ \\
\hline
\end{tabular}

Tableau 2 : séances CE2.

\begin{tabular}{|l|l|l|}
\hline $\begin{array}{l}\text { Contenu des } \\
\text { séances CE2 }\end{array}$ & $\begin{array}{l}\text { Activité des élèves } \\
(13 \text { filles et } 10 \text { garçons })\end{array}$ & Objectif de la séance \\
\hline
\end{tabular}




\begin{tabular}{|c|c|c|}
\hline Séance 1 & $\begin{array}{l}\text { répondre à un questionnaire portant } \\
\text { sur les langues autres que le français } \\
\text { parlées dans la famille des élèves }\end{array}$ & $\begin{array}{l}\text { prendre conscience de la richesse des } \\
\text { contacts linguistiques évoqués et la } \\
\text { diversité des langues présentes dans la } \\
\text { classe }\end{array}$ \\
\hline Séance 2 & 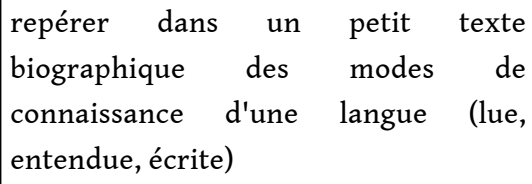 & $\begin{array}{l}\text { prendre conscience des langues que } \\
\text { l'on a entendu parler et des langues que } \\
\text { l'on a vu écrites }\end{array}$ \\
\hline Séance 3 & $\begin{array}{l}\text { classer les jours de la semaine en trois } \\
\text { langues (français, une langue } \\
\text { alphabétique et une langue à } \\
\text { idéogrammes) }\end{array}$ & $\begin{array}{l}\text { développer une stratégie de déduction } \\
\text { et d'inférence }\end{array}$ \\
\hline
\end{tabular}

Les séances ont fait l'objet d'un enregistrement audio afin d'analyser les interactions élèves-enseignante et élèves-élèves. Dans certains cas, les interactions à l'intérieur des groupes de travail ont été enregistrées. À ce stade de l'étude, il n'y a pas eu d'entretiens avec les élèves, leur réflexion n'étant pas suffisamment aboutie. Pour des commodités de lecture, nous faisons apparaitre pour chaque savoir l'analyse des interactions en CE1 puis celles de CE2.

\section{Analyse des données}

\subsection{Traces de développement de savoir-être}

L'analyse des interactions permet d'identifier une ouverture à l'autre. Ces traces sont peu nombreuses, bien que significatives sur leur potentiel de développement d'une compétence interculturelle.

En CE1, on ne trouve que quatre exemples de jugements de valeur sur les langues et les pays :

Émeline $Y$ a la langue française.

Maîtresse Ah, la langue française, le français tout de même! On le parle tous les jours.

Nicolas Aussi il y a l'allemand.

Baptiste Tout le monde connaît le français.

Maîtresse Quoi d'autre?

Alexandra L'espagnol.

Autre voix Ah, oui, ah j'adore l'espagnol.

Nicolas Moi je sais bien dire les noms en espagnol.

Une voix Le russe.

Maîtresse Le russe.

Jules C'est quoi le russe ? [...]

Jules La Russie. La Russie c'est le plus grand pays.

Jules se pose la question, non explicitée, de la nature du russe puis donne une information factuelle sur la Russie. Plus tard, quelqu'un dit que " Tahiti c'est très beau ". 
ces traces sont encore moins nombreuses. La maitresse demande à Jordan, qui a entendu parler des Néo-Zélandais et un Australien, si leur langue est "très différent [e] de la nôtre". Jordan se contente de répondre par l'affirmative "oui" et passe immédiatement à un autre sujet.

De même, lorsque la maîtresse essaie de faire émerger les opinions sur les systèmes d'écriture, les enfants restent très neutres dans leurs appréciations :

Maîtresse Où est-ce que tu as vu écrit du japonais?

Manon Euh, [inaudible]

Maîtresse Et alors, comment on sait que c'est du japonais, ou du chinois?

est-ce que cela ressemble à ce qu'on a nous? Nicolas.

Nicolas Eh bé, parce que ils écrivent pas comme nous. Il y en a c'est une fenêtre, euh...

Maîtresse Oui, on peut dire que ça ressemble un peu à des ...

Nicolas A des signes. [...]

Perrine Quand je suis allée en Arabie avec mes parents, on avait des papiers, alors, il y avait de l'arabe.

Maîtresse Ah, ça c'est intéressant, alors, qu'est-ce que tu as vu de différent par rapport au français ? cela t'a paru semblable, différent?

Perrine Euh différent.

Alors que "Tout le monde connaît le français" relève d'un stéréotype qui peut se révéler négatif dans l'ouverture à l'autre, il semble que la neutralité des enfants à l'égard des dissemblances avec la langue/culture maternelle révèle une acceptation de la différence sur laquelle l'enseignement peut s'appuyer. De même les clichés positifs, les réflexions comme "j'adore l'espagnol" ou "Tahiti c'est très beau" ne témoignent pas d'une prise de position marquée. Il s'agit plutôt d'opinions toutes faites qui ne semblent pas engager l'affect de l'auteur.

À cet égard, l'exemple ci-dessous est particulièrement pertinent: Elsa a passé ses vacances en Thailande où elle a rencontré une petite Italienne et une petite Portugaise. Elle communiquait avec elles en anglais dont sa mère lui avait fait apprendre quelques rudiments pour se faire comprendre à l'étranger. Elle raconte qu'elle a appris quelques mots de thaï :

Elsa L'année dernière j'étais allée en Thaïlande, aux grandes vacances et en fait, on est allé à la plage et je me suis fait deux copines, une Italienne et une Portugaise. [...]

Maîtresse Et alors, toi comment tu as parlé avec elles?

Elsa Euh anglais. Et il y en avait une autre ...[...] Il y en avait une qui habitait en Thaillande, .... Eh bien pareil, elle parlait thaillandais mais je leur parlais anglais.

Maîtresse D'accord. Et est-ce que des fois tu comprenais certains mots qu'elle te disait dans leur langue d'origine?

Elsa Oui, j'ai appris à dire (mot en thaï inaudible)

Autre voix C'est quoi?

Elsa (traduction inaudible)

Maîtresse Ben c'est déjà pas mal.

Elsa Et maîtresse ...

Maîtresse Oui?

Elsa En fait, c'est bizarre, parce que les mots des garçons, c'est pareil sauf qu'il y a "p" à la fin. Par exemple, si on veut dire bonjour et que c'est un garçon, alors c'est "sawadikap" et, pour les filles c'est "sawadika". 
Maîtresse D'accord. Donc, ça veut dire qu'on ne dit pas la même chose à une fille ou à un garçon pour dire bonjour. Et pour tous les mots est-ce que tu sais si c'est toujours un "p" à la fin?

Elsa Oui.

44 Aux deux niveaux, la cohabitation de langues/cultures différentes ne semble pas perturber les élèves. Cela reflète la tolérance à la différence propre aux jeunes enfants, ainsi que O'Neil (1993) le remarque. Elle souligne que les enfants, dès 4 ans, font des commentaires à propos de leurs problèmes de compréhension de la nouvelle langue et de ses caractéristiques "c'est différent", "c'est bizarre", "c'est rigolo" (Laura CE2). Les réflexions montrent qu'ils comprennent que les deux langues sont différentes et cette compréhension elle-même révèle l'émergence de la fonction métalinguistique. Cela est à rapprocher de l'absence d'inhibition devant l'expression orale en langue étrangère. Cette absence d'inhibition permet à l'enfant de s'exprimer avec une grande spontanéité mais également de ne pas prêter attention au code oral utilisé.

D'ailleurs, Marie n'hésite pas à faire des gestes pour se faire comprendre :

Maîtresse Tu t'es fait une copine anglaise, et comment vous avez parlé entre

vous?

Marie On a parlé anglais ...

Maîtresse Tu as parlé anglais?

Marie Mais s'il y avait des mots que je ne connaissais pas, je parlais avec des

gestes ... (rires dans la classe)

Autre exemple, lorsque la maîtresse s'étonne de ce que le père de Mathieu ait pu parler avec des Japonais, Mathieu, quant à lui, ne s'est pas rendu compte que la communication avait dû être difficile :

Mathieu Et mon papa il a demandé au Japonais et il a dit xxxx [inaudible]

Maîtresse Et le Japonais il comprenait le français? Comment il a parlé, papa,

au Japonais?

Mathieu Euh je sais pas.

47 L'enfant est beaucoup plus prêt que ne l'est l'adulte à accepter les différences, la nouveauté, le changement (Trocmé-Fabre, 1991). Cela est en partie dû au fait que l'enfant est engagé dans la découverte du monde, aussi bien physique que social et des règles qui le régissent. Mais cet apprentissage n'est pas encore figé et cela permet à l'enfant d'accueillir une autre langue /culture sans réticence.

\subsection{Traces de savoir-faire}

Voyons à présent ce que cette souplesse cognitive permet dans le domaine de l'acquisition de savoir-faire, c'est-à-dire dans la construction de stratégies d'inférence.

Lors de la séance consacrée à la découverte des jours de la semaine en trois langues, les enfants sont interrogés sur les stratégies qu'ils ont mises en œuvre pour découvrir de quels mots il s'agit, de la façon de les classer ensemble, et de quelles langues sont représentées.

On donne les noms des jours de la semaine en trois langues (dont le français) à des équipes de trois ou quatre élèves. La consigne est de classer ces mots en trois groupes, sans que soit donné tout autre renseignement. On constate que les enfants se mettent à la tâche sans demander d'autres explications. Sur les 4 groupes de CE1 et 5 groupes de CE2, 
il a été nécessaire de ré-expliquer les consignes à un seul d'entre eux. On voit très rapidement que les enfants ne s'interrogent pas sur la nature des mots qu'on leur donne. À partir du moment où ils disposent des noms des jours en français, ils en déduisent que les autres mots représentent aussi les jours de la semaine, comme le disent Pierre et Vincent :

Maîtresse Alors, tu crois que c'est les jours de la semaine? Alors comment tu le sais?

Pierre Parce que, ben, le, la langue français, c'est les jours de la semaine.

Maîtresse Ah, d'accord.

Pierre Alors, peut-être que les autres c'est des, c'est encore les jours de la semaine ...

Vincent En d'autres langues.

Ils s'appuient sur des stratégies d'inférence pour classer les mots par langue. Ils commencent par mettre de côté les mots issus de langues à idéogrammes (hébreu, arabe et chinois) dont ils savent d'instinct qu'ils ne pourront pas les lire et donc les classer. Là encore se constate leur tolérance à l'inconnu. Une fois interrogés sur cette mise de côté, ils déclarent tout simplement : "Alors, on a, là on a mis au hasard parce qu'on ne sait pas ce que ça veut dire.", "Non, nous on les a mis au pif. On peut pas savoir", "On l'a mis au hasard.", "Et ceux-là alors? Ben ceux-là on sait pas. ". Il est remarquable que les enfants de CE2 sont les seuls à verbaliser sur ce choix, alors que les élèves de CE1 n'en parlent pas. Pour quelle raison? On peut penser qu'ils ne sont pas déroutés par ces langues sans que cela ait besoin d'être exprimé, ou bien qu'ils sont trop occupés à trier l'autre liste pour s'en occuper.

Les groupes sont ensuite interrogés sur les méthodes utilisées pour classer les mots dans l'ordre. Signalons que la consigne était de classer les mots en trois listes mais pas de les mettre dans un ordre quelconque. Or, d'instinct, tous les groupes ont cherché à mettre les noms des jours dans l'ordre en cherchant des similitudes avec le français. Leur point de référence est le nombre de mots pour la semaine :

Michèle Alors, qu'est-ce qui ... quel est l'indice qui pourrait nous dire que c'est les jours de la semaine?

Jules $\mathrm{Y}$ a sept mots, là y a sept mots

Tous ont réussi à mettre les jours dans l'ordre à l'exception d'un seul en CE1 qui a confondu le mot français "lune" avec le mot espagnol pour lundi "lunes" :

Émeline Non, c'est " lune ".

Maîtresse Qu'est-ce que c'est que cette histoire de lune?

Émeline Ben on a mis lune (=Lunes), parce que on le dit en français, lune. [...]

Jules Ben, non parce que c'est "luné" ( =lunes), c'est "luné", c'est pas "lune".

J'ai compris pourquoi on a fait cette erreur, ça c'est dans cette langue-là parce que "lune" (=Lunes) ça veut dire lundi, euh, "marte" ça veut dire mardi, ça, ça veut dire mercredi, ça, ça veut dire jeudi, ça, ça veut dire vendredi, ça, ça veut dire samedi, et "domingo", ça veut dire dimanche.

Pour déterminer les groupes de mots, deux groupes se sont intéressés à la fin des mots :

Maîtresse Ah, c'est de l'anglais?

Christopher Oui. [...]

Autre voix Oui, c'est pareil à la fin, le même son.

Maîtresse Ah, c'est le même son? 
Autre voix Oui, ay, ay, ay, ay

Maîtresse Eh bien oui.

Autre voix Partout ay!

Maîtresse Dans l'autre groupe, ça fait Friday Tuesday Thursday Sunday,

Wednesday, Saturday, Monday. Donc?

Autre voix C'est pareil.

Ou bien pour l'allemand :

Michèle Alors, je vais vous les dire, Dienstag Donnerstag Samstag Montag Mittwoch Freitag Sonntag.

Plusieurs voix Tag, tag, tag

Autre voix On entend tag. généralement utilisée par les CE1: "Non, c'est pas en français, parce qu'il y a écrit VENERDI, mais c'est vendredi parce qu'il y a le V-E-R-D-I de vendredi.". Ou bien encore "Ben parce que c'est lundi, parce que il y a L-U-N et le D-I de lundi, il y a le M-A-R-D-I de mardi, il y a le M-D-I, et le C-I et le $R$ de mercredi ...".

61 En CE2, par contre, les lettres de début de mots permettent d'identifier Dimanche / Domingo : "Parce que ça commence par un D, là c'est mardi, parce que ça commence par un $M$, là c'est mercredi parce que ça commence par un $M^{\prime \prime}$.

C'est cette stratégie qui leur a permis de distinguer mardi et mercredi en italien :

Maîtresse Ah, oui, alors regardez, ensuite comment vous avez fait pour savoir que c'est mardi, martedì ? Mathieu?

Mathieu Parce que ça ressemble à mardi aussi. Il y a le $\mathrm{M}$, le R et le I.

Maîtresse Et ensuite avec mercoledì. Tout le monde pense que c'est mercredi, pourquoi ? Pauline?

Mathieu Parce qu'il y a un $M$ au début et ... 
Maîtresse Pourquoi ce serait pas mardi alors?

Mathieu Parce qu'à mercoledì, il y a M-E-R et qu'à martedì, il y a M-A-R.

Jules Ça doit être jeudi parce que .../

Hugo .../ Parce que des fois le $G$, parce que des fois le $G$ ça fait $[j]$, parce que dans girafe, y a un $G$ qui fait [j].

64 Rappel du patrimoine linguistique et culturel : un groupe de CE1 a fait appel à ses connaissances de la langue étrangère sans que l'on puisse déterminer d'où venait cette connaissance :

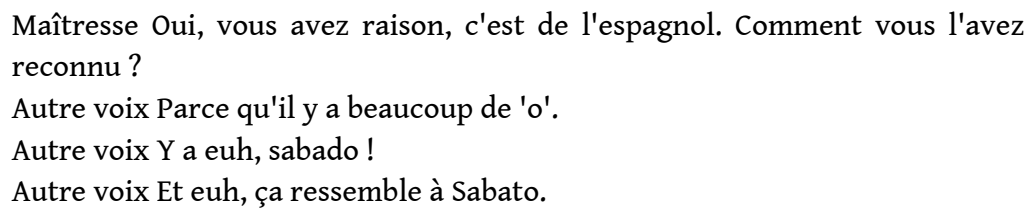

5 De nombreux groupes ont fait appel à leur patrimoine culturel, qu'il soit issu de voyages personnels, d'origines familiales, de connaissances familiales partagées ou de leur culture propre :

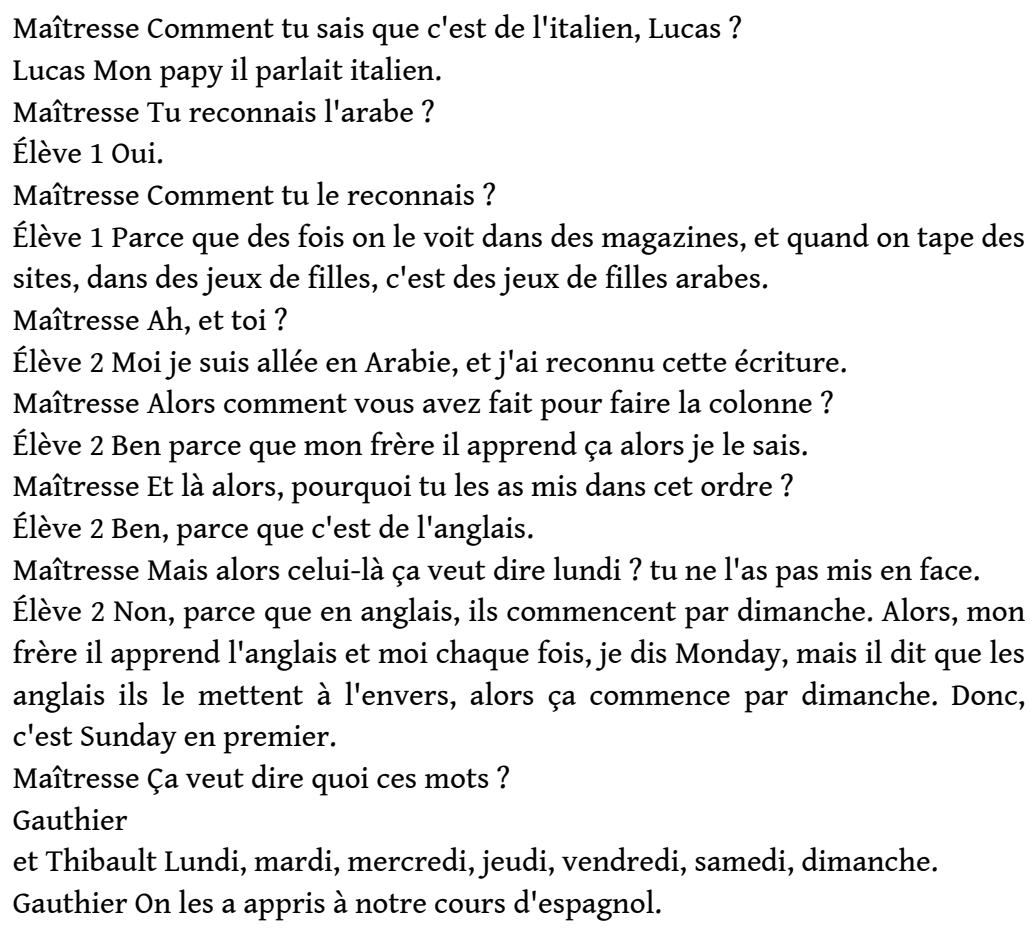

Tous ces exemples montrent bien que les enfants n'arrivent pas en classe vierges de toute connaissance et même s'il s'agit de leur première expérience d'apprentissage d'une langue étrangère, on est étonné par la richesse de leur patrimoine culturel sur lequel l'enseignant de langue étrangère peut appuyer la sensibilisation interculturelle. Même s'il s'agit de connaissances parcellaires, on ne peut les ignorer, elles sont présentes et peuvent représenter un point d'appui sur lequel l'action didactique pourra s'exercer.

De même, on trouve les appuis à une véritable compétence métalinguistique lorsque les élèves comparent le français et le thaï : 
Maîtresse On n'a pas quelque chose quand même qui différencie les filles et les garçons en français? réfléchissez, est-ce qu'il n'y a pas ...

Voix Le mascu...

Maîtresse Réfléchissez.

Voix Si, je sais ...

Maîtresse Iris?

Iris Le masculin et le féminin.

Maîtresse Et alors Iris, qu'est-ce qui différencie dans la langue le masculin et le féminin?

Iris Le déterminant. étrangère très restreints mais les CE2 ont quelques connaissances essentiellement lexicales provenant soit des connaissances familiales soit de leurs expériences (la tenue de matches de la Coupe du Monde de Rugby dans la région a grandement contribué à étendre la culture de nos élèves !) :

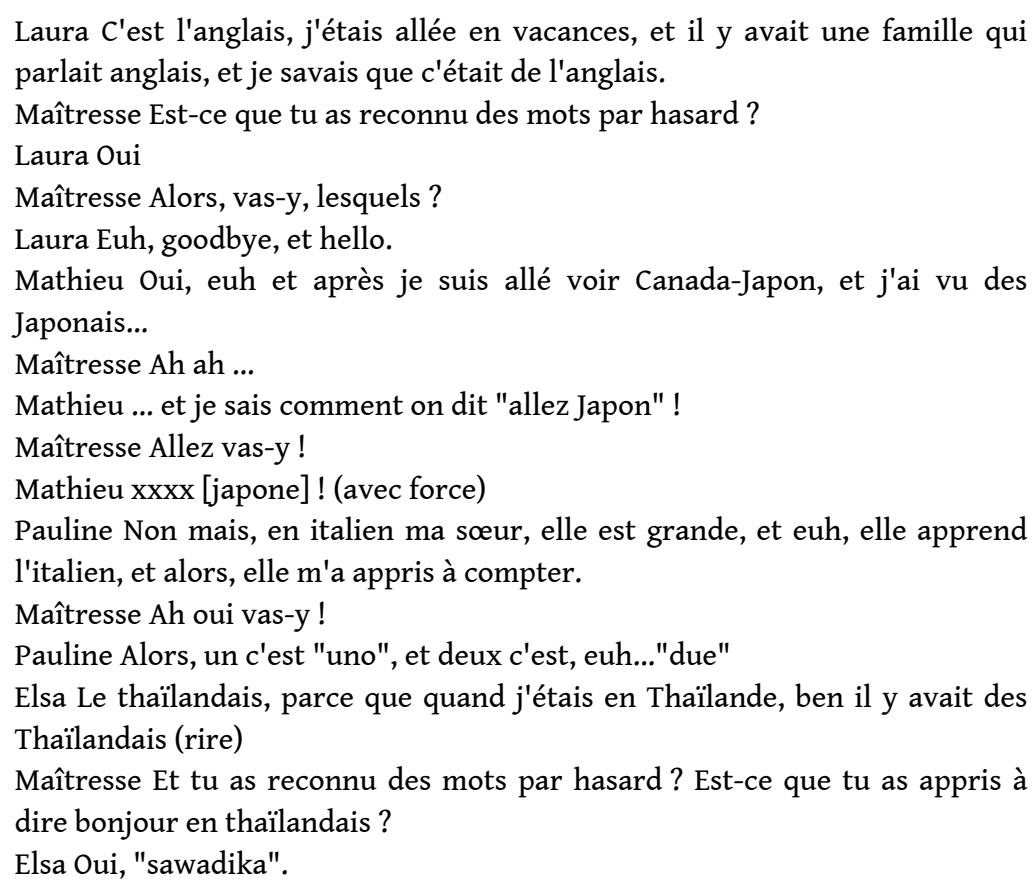

71 Ces connaissances ne sont pas exploitables en tant que telles mais la langue comme moyen véritable de communication, elles contribuent à renforcer sa légitimité en contexte scolaire. Par contre, les contacts interethniques favorisés par nos sociétés multiculturelles ne doivent pas être négligés et les nombreux exemples relevés dans les séances en sont la preuve. Au cours de la séance où les enfants nomment les langues qu'ils ont entendu parler, il est clair que la vie actuelle d'un enfant donne des occasions de rencontres avec des locuteurs d'autres langues que la langue dominante du pays : 
Jordan Mais il y a à côté de chez moi, mon voisin, il parle d'autre langue(s).

Perrine Mes cousins anglais. Ils parlent anglais

Mathieu Moi, mon petit cousin de un an, euh deux ans. Il parle anglais.

Maîtresse Il parle anglais ? pourquoi parle-t-il anglais?

Mathieu Avec les Chinois, parce qu'il vit en Chine.

Nicolas Marius, il parlait français mais je crois qu'il parle espagnol. Il vit en

Espagne, son père et sa mère ils parlent espagnol.

Pauline Moi j'étais allé en Bretagne et j'avais rencontré des Belges. Je connais

un mot en belge. On était en camping.

Jordan Le petit que ma mère garde, il est de Colombie.

Maîtresse Et il parle comment, ses parents ils parlent comment?

Jordan Ben, exactement colombien, moi je sais pas trop comment il parle ...

Les exemples abondent pour montrer que la séance de sensibilisation à l'interculturel passe par leur vécu et permet de faire émerger ce patrimoine passif et inconscient. C'est à partir de ces confrontations que les élèves peuvent envisager l'utilité de l'apprentissage d'une langue étrangère.

\section{Traces de savoir-apprendre}

Les enfants susceptibles de développer des savoir-apprendre sont capables de créer et d'établir des liens entre leur expérience de ce qu'est une langue, et de sa fonction de communication. Ainsi à la question, "qu'est-ce qu'une langue? ", un enfant de CE1 parle de l'organe dans la bouche :

Tanguy Elle est là !

Maîtresse Ah, elle est là la langue ! oui.

Autre voix Elle est attachée !

Maîtresse Oui, elle est attachée. C'est le même mot?

Une voix Oui. On peut pas l'enlever. Elle est attachée.

Maîtresse Donc, pour parler une langue ...

Élise ... il faut avoir une langue.

La langue sert à "parler", "comprendre" "comprendre ce que dit l'autre", "communiquer", " parler", "lire des histoires", "pour se repérer". D'autres encore signalent qu'une langue "c'est un langage", "des mots", "des phrases", "des chansons", "des poésies".

Élise pense aux sourds-muets, elle fait ainsi la preuve que pour elle, la langue est un moyen de communication avant tout. Pourtant, cela ne fait pas l'unanimité chez ses camarades car Alexandra pense que "Si on veut parler il faut de la voix!". Mais cela n'est pas aussi certain pour ses camarades :

Élise Il y en a qui font des gestes. Il y en a qui font des gestes.

Maitresse Alors, explique nous, tu veux nous parler de quelles personnes?

Élise Des personnes qui ne parlent pas.

Maîtresse Ah, d'accord.

Autre voix Les sourds-muets!

Maîtresse Alors, ceux qui sont muets, ceux qui parlent avec les gestes, qu'est-

ce que tu m'as dit, ce n'est pas ...

Élise Ce n'est pas une langue.

Maitresse Ah ce n'est pas une langue?

Pierre Si, c'est une langue ! c'est une langue pour parler.

Maîtresse Oui, Pierre?

Pierre C'est une langue !

Maitresse Oui, comment elle s'appelle cette langue?

Pierre Euh ...la langue des gestes. 
". Ils se mettent finalement d'accord pour dire qu'il s'agit d'"une façon de parler". On voit bien à travers ces exemples que la notion d'apprentissage d'une langue est loin d'être claire. Il s'agit en général pour eux de savoir quelques mots, de se faire comprendre même avec les gestes.

Le savoir-apprendre se développe également par la capacité à s'interroger sur les stratégies mises en œuvre pour établir la compréhension, comme dans l'exemple suivant (CE1) :

Lucas C'est de l'italien. [...] Sabato, c'est samedi.

Maîtresse Comment tu sais que c'est de l'italien, Lucas?

Lucas Mon papy il parlait italien.

Michèle Il est italien ton papy?

Lucas Il est né en Italie. [...]

Jules Moi j'ai une question pour Lucas.

Maîtresse Alors une question pour Lucas.

Jules Comment tu peux savoir que ton papy, il parle italien alors que tu connais pas cette langue?

Lucas Euh ...

Jules Et comment tu peux le comprendre quand il te dit bonjour, au revoir, merci ...

Lucas Parce qu'en fait quand il était petit, il était en Italie jusqu'à trois ans, et il s'en rappelait plus. Il était né en Italie et après il a déménagé en France.

Jules Et maintenant, tu comprends ce qu'il dit. Parce que maintenant il parle la langue française.

Les enfants ne distinguent pas entre la nature et la fonction de la langue mais comprennent bien qu'elle est nécessaire pour se comprendre et communiquer.

Leurs voyages personnels ou ceux dont ils ont pris connaissance par l'intermédiaire de leur entourage permettent également aux élèves de créer des passerelles entre leur expérience de l'usage d'une langue et leur apprentissage actuel. C'est ainsi que se crée le lien avec l'enseignement de la langue dans leur classe, bien que le choix de la langue ne soit pas aussi clair que l'on pourrait le penser. En effet, la réponse que nous tentions de faire émerger était la nécessité d'apprendre une langue commune afin de favoriser la compréhension mutuelle.

81 Or, nous avons observé que la pertinence de la langue de communication commune est loin de s'imposer dans leur esprit. À la question: "Est-ce que vous, vous trouvez intéressant de parler d'autres langues ?", les enfants de CE2 répondent positivement mais ne semblent pas percevoir la difficulté d'apprendre les langues de tous leurs interlocuteurs, comme en témoignent les exemples ci-dessous :

Simon Si on va en Italie, par exemple, on trouve un copain, on peut lui parler par exemple ... on peut lui parler portugais ...

Alex Si on va en Italie, par exemple, si on se trouve un copain, et qu'on apprend pas à parler italien, on comprend pas quand il parle italien, on peut pas et l'autre jour à la plage, moi et mon frère on s'est trouvé un copain, et il parlait anglais.

Estelle Quand t'es adulte, tu peux aller en vacances, on peut parler...

Laura Je trouve que c'est bien. Parce que c'est rigolo de parler avec des gens qui parlent. 
Mais un autre affirme :

Autre voix L'espagnol, parce que tous les mois je vais en Espagne Maîtresse Ah toi tu veux apprendre l'espagnol parce que tu vas en Espagne. Baptiste L'anglais ... parce qu'il y a plus de monde qui parle anglais, que des gens l'espagnol.

\section{Conclusion}

Ces premières séances nous ont permis de vérifier l'émergence des composantes d'une prise de conscience interculturelle conformément au cadre défini par Byram. Pour embryonnaires qu'elles soient, nous y voyons les bases de la construction d'une compétence qui ne serait pas seulement réduite à des connaissances mais qui reposerait plutôt sur des habiletés et des attitudes à développer. S'il est très difficile de mesurer les acquisitions dans ce domaine, il semble cependant souhaitable de placer l'apprenant en activité sur un objet culturel pour révéler les représentations de l'autre et les affiner. Les représentations des élèves sont de deux ordres : des représentations d'ordre personnel relatives à l'enfant en tant que personne et en tant qu'apprenant, et des représentations relatives à une vision de l'altérité telle qu'elle peut être véhiculée par la langue/culture cible. Ces représentations ne sont pas nécessairement des obstacles à l'apprentissage mais nécessitent d'être reconnues de l'enseignant en tant que telles. L'intérêt de ces activités de sensibilisation est de provoquer une réflexion métacognitive de la part des apprenants tout en conférant à l'enseignant des outils d'ajustement de ses interventions au cours d'interactions avec le groupe. En effet, la comparaison de tous les éléments constitutifs de la langue est une démarche interculturelle globale où l'enseignant soumet à ses élèves des images de l'Autre et des images du Même. Ces dernières représentent des images de ce qui est connu et familier, souvent des images concernant la culture source. Les images de l'Autre font référence à ce qui est étranger et inconnu et sont souvent mises en rapport avec la culture cible.

Bien des pistes restent à explorer à l'issue de cette première phase de recherche. En référence à la compétence de "médiation" définie par le CECRL comme le fait de "produire à l'intention d'un tiers une (re)formulation accessible d'un texte premier auquel ce tiers n'a pas d'abord accès direct" (CECRL, 2001 : 18), nous substituons la notion 
de "médiateur culturel" définie par Zarate et al. (2003) en considérant chaque locuteur comme un passeur de culture. Il nous paraît important de développer chez les apprenants, dès le plus jeune âge, la capacité d'interpréter et de mettre en relation, d'utiliser des connaissances existantes pour comprendre un document spécifique ou un comportement par exemple, et de mettre en relation ce document ou ces comportements avec d'autres comparables dans son groupe social. À cet effet, on pourra utiliser les Tuic ${ }^{1}$ pour mettre les élèves en contact avec la langue et avec ses locuteurs. Cette option permettra de se pencher sur les aspects collaboratifs d'une telle pratique pour développer des moments de réflexion et favoriser une ouverture interculturelle. L'objet d'une telle pratique est de développer des compétences d'ordre linguistique et social en s'appuyant sur le rôle d'acteur social de chaque apprenant, relationnel en développant les capacités à entrer en contact avec la langue/culture cible, discursif en les encourageant à utiliser différentes modalités d'expression, et socioculturel pour favoriser le décentrage par rapport à la langue/culture maternelle.

\section{BIBLIOGRAPHIE}

Abdallah-Pretceille M. (1996). Vers une pédagogie interculturelle. Paris : Anthropos.

Abdallah-Pretceille M. (1999). L'éducation culturelle. Paris : PUF.

Bulletin Officiel de l'Éducation Nationale, Hors Série, $n^{\circ} 1$ du 14 février 2002. http:// www.education.gouv.fr/bo/2002/hs1/default.htm

Byram, M. (1997). Teaching and Assessing Intercultural Communicative Competence. Clevedon : Multilingual Matters Ltd.

Byram, M. \& Zarate, G. (1998). "Définitions, objectifs et évaluation de la compétence socioculturelle". In Apprentissage et usage des langues dans le cadre européen. Le français dans le monde - Recherches et applications, pp. 70-96. Paris : Hachette et EDICEF.

Byram, M., Zarate, G., \& Neuner, G. (1997). La compétence socioculturelle dans l'apprentissage et l'enseignement des langues. Vers un Cadre européen commun de référence pour l'apprentissage et l'enseignement des langues vivantes : études préparatoires. Éditions du Conseil de l'Europe. Cadre européen commun de référence pour les langues (2001). Paris : Didier.

Galisson, R. \& Puren, C. (1999). La Formation en questions. Paris : CLE International.

Genelot, S. (2001). "Evlang : bilan de trois années de recherches sur l'éveil aux langues dans l'enseignement primaire". http://www.u-bourgogne.fr/LABO-IREDU/2001/01113.pdf.

Kervran, M. (2006). Les langues du monde au quotidien. Observation réfléchie des langues. Cycle 2 et 3. Rennes : Scérén/CRDP Bretagne.

Luc, C. (1992). "Des représentations aux productions en langue étrangère dans le cadre scolaire". Repères, $\mathrm{n}^{\circ} 6$ pp. 23-40.

Macaire, D. (1998). "L'éveil aux Langues à l'école primaire en contexte européen : éléments de réflexion pour la formation des enseignants". In Billiez, J. (dir.). De la didactique des langues à la 
didactique du plurilinguisme. Hommage à Louise Dabène. Grenoble : CSL-Lidilem, Université Stendhal- Grenoble III, pp. 341-353.

Mattar, C. \& C. Blondin (dir.). (2003). S'ouvrir aux langues et aux cultures grâce à l'éveil aux langues. Université de Liège : Service de Pédagogie expérimentale.

Moore, D. (2001). Les représentations des langues et de leur apprentissage. Paris : Didier.

O'Neil, C. (1993). Les enfants et l'enseignement des langues étrangères. Paris : Hatier/Didier : Langues et apprentissage des langues.

Pross, N., Gaux, C. \& Gaonac'h, D. (2002). "Évaluation du centre exécutif de la mémoire de travail chez de jeunes enfants de cycle II et III". Colloque : Développement cognitif et troubles des apprentissages : évaluer, comprendre, rééduquer et prendre en charge (12-13 décembre 2002). Strasbourg : France.

Trocmé-Fabre, H. (1991). J'apprends, donc je suis. Paris : Les Éditions d'Organisation.

Zarate, G. (2001). "Les compétences interculturelles : définition, place dans les curriculums". Actes du séminaire: L'enseignement des langues vivantes, perspectives. (25 octobre 2001). http:// eduscol.education.fr/D0033/langviv-acte6.htm.

Zarate, G., Gohard-Radenkovic, A., Lussier, D. \& Penz, H. (2003). Médiation culturelle et didactique des langues. Strasbourg : Éditions du Conseil de l'Europe.

\section{Logiciel}

Auger, N. (2005). Comparons nos langues. Démarche d'apprentissage du français pour les enfants nouvellement arrivés. Cédérom "Ressources Formation Vidéo Multimédia". Scérén / CRDP académie de Montpellier.

\section{NOTES}

1. Vocable remplaçant celui de Tice pour désigner les «Technologies Usuelles de l'Information et de la Communication».

\section{RÉSUMÉS}

Cet article vise à analyser le potentiel de séances de sensibilisation aux langues / cultures au cours des toutes premières séances de langue étrangère au cycle 1. Les interactions en classe ont été analysées selon le modèle de Byram afin d'identifier les composantes d'une prise de conscience métalinguistique. Nous soulignerons les éléments essentiels d'une telle approche dans la construction d'une compétence interculturelle qui ne reposerait pas seulement sur des connaissances mais plutôt sur le développement de savoir-être et de savoir-faire.

This article aims at analysing the potential of raising language and culture awareness during the very first lessons of foreign language teaching in years 2 and 3. Classroom interactions have been analysed according to Byram's model so as to identify the components of a metalinguistic 
process. We will highlight the key elements of such an approach in the construction of an intercultural competence which would not only rely upon knowledge but rather upon the development of existential competence, skills and know-how.

\section{INDEX}

Mots-clés : démarche interculturelle, apprentissage précoce de l'anglais, savoir-être, savoirfaire, savoir-apprendre

Keywords : intercultural process, early English learning, existential competence, skills, ability to learn

\section{AUTEUR}

\section{MICHĖLE CATROUX}

Michèle Catroux est maître de conférences en Didactique de l'Anglais au Laboratoire ASPDA Université Victor Segalen Bordeaux 2. Son domaine de recherche est principalement centré sur l'enseignement /apprentissage de l'anglais à l'école primaire. Elle étudie les effets des modes collaboratifs d'apprentissage médiatisés par les Tuic. Elle travaille actuellement sur la construction de la compétence interculturelle à des niveaux précoces d'apprentissage en partenariat avec des Professeurs des Écoles en exercice.

Courriel : michele.catroux[at]aquitaine.iufm.fr Adresse : IUFM d'Aquitaine, Université Montesquieu - Bordeaux 4, 49 rue de l'École Normale, BP 210, 33021 BORDEAUX, France. 